\title{
Challenges of Mass Casualty Management at a Regional Trauma Centre in Sub-Saharan Africa
}

\author{
Muhammad Oboirien \\ Department of Surgery, Usmanu Danfodiyo University Teaching Hospital, Sokoto, Nigeria \\ Email: moboirien@yahoo.com
}

Received 10 December 2013; revised 20 January 2014; accepted 29 January 2014

Copyright (C) 2014 by author and Scientific Research Publishing Inc. This work is licensed under the Creative Commons Attribution International License (CC BY). http://creativecommons.org/licenses/by/4.0/ CC) (i) Open Access

\begin{abstract}
Background: Mass Casualty incidents usually overwhelm the capabilities of any centre. It is an event whose nature is undetermined, unexpected and disrupts the normal trauma care in a hospital. Hospitals have established protocols to deal with mass casualty when it arises. Objectives: We sought to profile the presentations of mass casualty incidents and challenges in management in a regional trauma Centre in North-West Nigeria. Methodology: A retrospective look at the records of mass casualty presentations in our centre over a 12-month period (January-December 2011) was done. A mass casualty event in our setting is the presentation to our facility of eight or more patients from the same cause at the same. Results: There were a total of 18 mass casualty presentations with an average of 1.5 per month. There were a total of 236 victims including those brought in dead (BID). Males were 203 (86\%) and females 33 (16\%) with an M:F ratio of 6:1. Majority of the victims $222(94 \%)$ were above 16 years and all the victims had a common mechanism of injury which was Road traffic crashes. One hundred and forty-three (61\%) of the victims sustained lacerations and bruises while $31(13 \%)$ were polytraumatized. Sixty-two (26\%) of the victims were BID'S from the scenes of the Road Crashes. The outcome was that 131 (75\%) of the victims were treated and discharged while 41 (24\%) were admitted for further management. Two (1\%) patients died during resuscitation and within 24 hours. Conclusion: Mass casualty presentations are a regular event in our centre hence there is the need for the establishment of a protocol and regular mass casualty drills to cope with future occurrences.
\end{abstract}

\section{Keywords}

Mass Casualty; Management; Trauma 


\section{Introduction}

A Mass Casualty Incident (MCI) can be defined as an incident that has produced more casualties than a customary response assignment can handle. Even in the best of centres, mass casualty incidents carry the potential of overwhelming the capabilities of even the most organized medical systems. A comprehensive and immediate demand is made upon resources, rendering even the initial in-hospital phase of minimal acceptable care extremely difficult to maintain [1]. Types of incidents that can produce mass casualties include, road traffic crashes, building collapse and terrorist attack. Civilian mass casualty events are regular occurrences in our environment and the ability to cope depends on the treatment facility, location and the level of staffing. In rural areas with limited resources, a mass casualty event may be two trauma patients, while in a large city the total may have to go beyond 50, or even 100, before the system's capability is exceeded. Weather, traffic conditions, and a host of other factors may influence what a mass casualty is in a given case. Road traffic crashes are the common causes of mass casualty in our environment [2]-[4]. The concept of Terror related mass casualty that had hitherto been seen in countries of the Middle East is fast becoming a norm in our community as a result of insurgencies by militant groups. Terror related mass casualty events pose a new challenge for trauma care and require consideration of a paradigm shift [5]-[8].

Mass casualty incidents usually lead to high mortality from lack of adequate pre-hospital care, lack of preparedness of health facilities and inadequate and effective triage. Attempts have been made to categorize mass casualty incidents into minor, moderate and major depending on the number of casualty and other variables. According to the Jos protocol, minor mass casualty represents 7 to 30 casualties present at the same time, while a moderate mass casualty is when 31 to 50 casualties are present to the facility and a Major mass casualty is when more than 50 casualties are present. This is also referred to as regional disaster [2]-[4].

Countries with adequate pre-hospital care and established pre-hospital trauma life support PHTLS and the Advance trauma life support ATLS guidelines have made remarkable progress in dealing with mass casualty incidents. PHTLS and ATLS guidelines have been strictly followed in Israel, resulting in admission of most major civilian trauma victims to trauma centres [7]-[9].

Mass casualty incident management is still an evolving field and there are no national or regional emergency preparedness plans in our sub region. Limited resources in terms of material, personnel and absence of a Prehospital care continue to pose a major challenge in the management of mass casualty. Ambulance services and Helicopter based rescue services in these traffic prone cities are equally lacking [10] [11].

Our regional trauma centre was established in January 2009 to cater for cases of acute trauma. It covers the North West geopolitical zone of Nigeria comprising three states with an estimated population of 11 million. This study aims to review mass casualty incidents management at a regional trauma centre in Sub-Saharan Africa to highlight the challenges in management.

\section{Methodology}

This was descriptive, retrospective look at the records of mass casualty presentations in our centre over a 12months period (January-December 2011) was done. A mass casualty event in our setting is the presentation to our facility of eight or more patients from the same cause at the same. Trauma records of mass casualty incidents' involving eight or more patients presenting to our facility was checked for sex and age, mode of transportation to hospital, cause and nature of injury sustained, of patients, treatment offered and outcome. Results are presented in table and figure.

\section{Results}

There were a total of 236 victims including those brought in dead (BID). Sixty-two (26\%) of the victims were BID'S from the scenes of the Road Crashes. A total of 18 mass casualty incidents with an average of 1.5 per month were recorded with the highest in June as shown in Figure 1. There was an average 13.1 casualty per incident with males constituting 203 (86\%) and females 33 (16\%) with an M:F ratio of 6:1. Majority of the victims 222 (94\%) were above 16 years and all the victims had a common mechanism of injury which was road traffic crashes. In all the incidents, patients were brought to the centre by men of the road safety commission and some other volunteers in make shift truck and ambulances with no facilities for first aid. There was no prior notification of the centre when cases are brought and the centre is usually alerted by sounds of blazing sirens. One hundred 


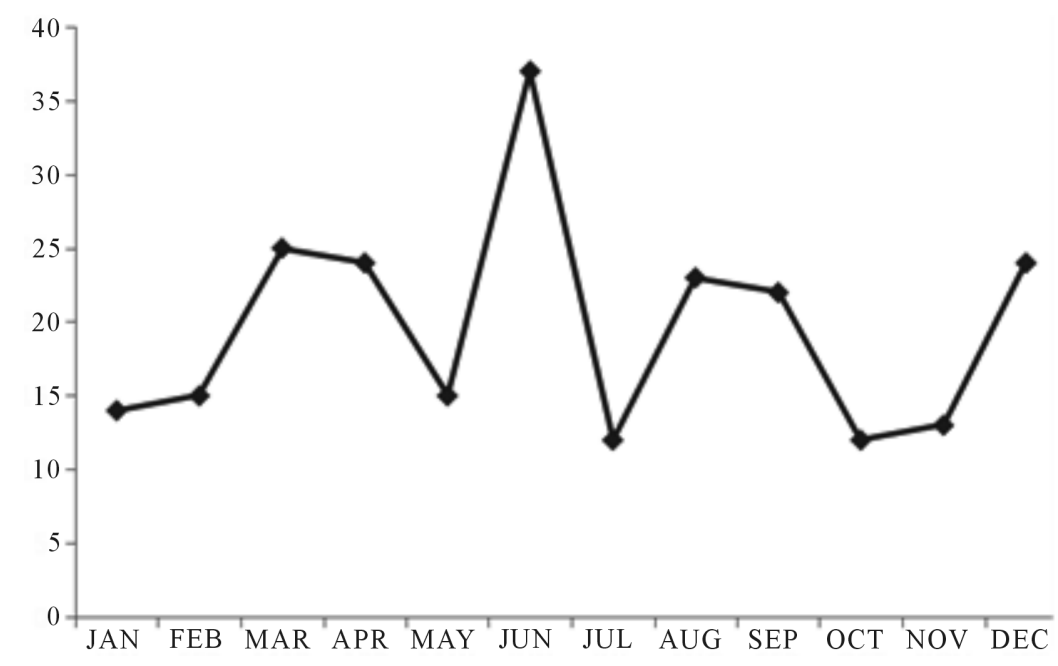

Figure 1. Line chart showing monthly distribution of casualties. Y-axis shows the number of victims per month.

and forty-three (61\%) of the victims sustained lacerations and bruises while 31 (13\%) were Polytraumatized. Sixty-two (26\%) of the victims were BID'S from the scenes of the road crashes with an average of 3.4 fatality per incidents as shown in Table 1. The outcome was that 131 (75\%) of the alive victims were treated and discharged while 41 (24\%) were admitted for further management. Two (1\%) patients died during resuscitation and within 24 hours.

\section{Discussion}

A total of 18 mass casualty incidents resulting in 236 casualties and fatality is quite a huge number for any centre to cope with. This number is aside the daily cases of trauma attended to and this is in spite of the poor resource and material availability. The trauma centre saw over 3000 patients the previous year with road traffic crashes being the commonest cause of trauma with $68.2 \%$ [12]. Vehicular transport serves as the common means of intercity mass transit for the majority of Nigerians. Efforts have been made by the Government to regulate these mass transits especially those that ply the roads at night but with little success. Mini buses which are used mainly for intercity travels are sometimes overcrowded with no regard for speed limit and safety, and these results in huge number of casualties and fatalities whenever there is a road crash [13]. The higher number of incidents and casualty seen in the second half of the year is due to the raining season which is intense during these periods and the high human and vehicular activities associated with religious festivities and intercity trades following harvest. Males were more affected as they tend to be involved in commercial and economic activities as the cultural norms of the people demands. Majority of the casualties are young individuals and these have been the victims of trauma in other studies and are more likely to die from trauma than any other cause [14] [15]. Our study noted that all the casualties were brought to the centre by men of the road safety commission and volunteers. This was done with no form of triage or any guideline as all casualties were brought together sometimes with fatalities. This underscore the near absence of pre-hospital care as any one injured is brought to the centre irrespective of the degree of injury. The number of those with laceration and bruises indicate that they could have been seen and treated at other health facilities if there was a proper and coordinated pre-hospital, while the centre is allowed to deal with more serious cases. This practice is in sharp contrast to the findings of Sharon et al. They noted that in the setting of terror related mass casualty events, the majority of all patients and the majority of urgent patients were evacuated to the nearest hospital and not necessarily to trauma centres [6]. This shows that even where there are designated trauma centres, most of the other hospital are still able to deal with mass casualty incidents unlike in our own scenario which puts pressure on one facility because of the ineffectiveness of others. The cases of polytrauma mainly involved fractures with head injuries or sometimes multiple fractures and these were appropriately managed by the respective specialties after stabilization. As noted, the presentation of more eight or more casualties at the same time from the same cause constitutes a mass casualty incident. Usually there is no prior notification before arrival, but once it happens the compound matron is notified and she 
Table 1. Nature of injuries and fatality.

\begin{tabular}{ccc}
\hline & NUMBER & $\%$ \\
\hline LACERATION/BRIUSES & 143 & 61 \\
POLYTRAUMA & 31 & 13 \\
BROUGHT IN DEAD & 62 & 26 \\
TOTAL & 236 & 100 \\
\hline
\end{tabular}

mobilises nurses from different wards to assist. The number so mobilized will depend on the magnitude of the incident and they are usually helpful in triage. The centre lacks infrastructures and high-tech imaging facilities, but it usually has resuscitation packs categorise as minor, intermediate and major packs available for use by patients but in event of incidents, the central pharmacy store is usually contacted to replenish and provide more stocks. Mass casualty management is government driven with little or no private sector investment. There are no funds earmarked for trauma care and no donor or non-governmental agencies are involved in trauma care compare to areas like HIV, malaria. The number of doctors usually required varies between 5 and 10 and these are represented by the casualty officers and the trauma team on duty. The trauma team comprise an orthopaedic surgeon, General Surgeon, Neurosurgeon and an Anaesthetic. Mobilisation and assemblage of the team is unpredictable and time consuming as the casualty officer have to rely on personal telephone contact. A closed group mobile phone system have been tried but with little success and resources has hampered the establishment of a pager system. Our study also noted a high rate of fatalities brought from the site and this shows the severity of most of the crashes and the lack of rescue when these crashes occur. As noted by Trunkey in his trimodal pattern of death, about half of trauma deaths will occur at the site of trauma or within the first hour from severe head and cardiovascular injuries [16]. Rescue and transport of patients to health facility is uncoordinated and the first responders are not trained to carry out appropriate and adequate triage. It is not unusual for patients to die of secondary brain injury. The study showed that that most of the casualties that made it to the hospital survived the first 48 hrs as we recorded two mortalities during resuscitation. Twenty-four per cent of patients were admitted for further management by the respective specialties and this study did not seek to find out the long term outcome with regards to those admitted. Further deaths cannot be ruled out as the third peak of trauma death can occur days or week later from complications of trauma and multiple organ failure [16]-[18].

\section{Conclusion}

Mass casualty presentations are a regular event in our centre hence there is the need for the establishment of a trauma system which has been defined as an organised approach to the acutely injured patient that is provided with personnel, facilities and equipment for optimal care on an emergency basis within a defined geographic area, and this begins right from the field. Almost $26 \%$ of the deaths were brought in dead, before any medical could reach them. This number would be significantly reduced if a trauma system is in place. Measures to reduce road crashes should be strengthened so as to reduce the number of mass casualty incidents. Health care facilities at all levels should equally be strengthened to meet the challenges of trauma care.

\section{Acknowledgements}

Our sincere appreciation goes to our support staff involved in the care of the injured.

\section{References}

[1] Hirshberg, A., Holcomb, J.B. and Mattox, K.L. (2001) Hospital Trauma Care in Mass Casualty Incidents: A Critical View. Annals of Emergency Medicine, 37, 647-652. http://dx.doi.org/10.1067/mem.2001.115650

[2] Nwadiaro, H.C., Iya, D., Yiltok, S.J. and Kidmas, A.T. (2003) Mass Casualty Management: Jos University Teaching Hospital Experience. West African Journal of Medicine, 22, 199-201.

[3] Nwadiaro, H.C. and Deshi, P.J.D. (1999) Concept of Mass Casualty Management in a Developing Country. The Nigerian Journal of Surgical Research, 1, 50-55.

[4] Nwadiaro, H.C. (1998) Principles and Protocol for mass Casualty Management in Jos University Teaching Hospital. JUTH Publication Series, Zimek Communication, Jos.

[5] Katz, E., Ofek, B., Adler, J., et al. (1989) Primary Blast Injury after a Bomb Explosion in a Civilian Bus. Annals of 
Surgery, 209, 484-488. http://dx.doi.org/10.1097/00000658-198904000-00016

[6] Sharon, E., Zvi, F., Charles, W., Daniel, Z., Guy, C., et al. (2004) Evacuation Priorities in Mass Casualty Terror-Related Events, Implications for Contingency Planning. Annals of Surgery, 239, 304-310. http://dx.doi.org/10.1097/01.sla.0000114013.19114.57

[7] Raiter, Y., Farfel, A., Lehavi, O., Goren, O.B., Shamiss, A., et al. (2008) Mass Casualty Incident Management, Triage, Injury Distribution of Casualties and Rate of Arrival of Casualties at the Hospitals: Lessons from a Suicide Bomber Attack in Downtown Tel Aviv. Emergency Medicine Journal, 25, 225-229. http://dx.doi.org/10.1136/emj.2007.052399

[8] Admi, H., Eilon, Y., Hyams, G. and Utitz, L. (2011) Management of Mass Casualty Events: The Israeli Experience. Journal of Nursing Scholarship, 43, 211-219. http://dx.doi.org/10.1111/j.1547-5069.2011.01390.x

[9] The National Association of Emergency Medical Technicians and the American College of Surgeons Committee on Trauma (2003) Introduction. In: McSwain, N. and Salomone, J., Eds., PHTLS: Basic and Advanced Prehospital Trauma Life Support, 5th Edition, Mosby, St Louis.

[10] Ehiawaguan, I.P. (2007) Mass Casualty Incidents and Disasters in Nigeria: The Need for Better Management Strategies. Nigerian Postgraduate Medical Journal, 14, 341-346.

[11] Adesunkanmi, A.R. and Lawal, A.O. (2011) Management of Mass Casualty: A Review. Nigerian Postgraduate Medical Journal, 18, 210-216.

[12] Oboirien, M., Ismail, S. and Agbo, P.S. (2011) Trauma Incidence in Sokoto, North-West Nigeria. Nigerian Journal of Orthopaedics and Trauma, 10, 105-109.

[13] Balogun, J.A. and Abereoje, O.K. (1992) Pattern of Road Traffic Accident Cases in a Nigerian University Teaching Hospital between1987 and 1990. The Journal of Tropical Medicine and Hygiene, 95, 23-29.

[14] Solagberu, B.A., Adekanye, A.O., Ofoegbu, C.P.K., Kuranga, S.A., Udoffa, U.S., Abdur-Rahman, L.O., et al. (2002) Clinical Spectrum of Trauma at a University Hospital in Nigeria. European Journal of Trauma, 28, 365-369. http://dx.doi.org/10.1007/s00068-002-1223-y

[15] WHO: Global Plan for the Decade of Action for Road Safety 2011-2020. www.who.int/roadsafety/decade_of_action/

[16] Trunkey, D.D. (1983) Trauma. Scientific American, 249, 28-35. http://dx.doi.org/10.1038/scientificamerican0883-28

[17] Solagberu, B.A., Adekanye, A.O., Ofoegbu, C.P., Udoffa, U.S., Abdur-Rahman, L.O. and Taiwo, J.O. (2003) Epidemiology of Trauma Deaths. West African Journal of Medicine, 22, 177-181.

[18] Baker, C.C., Oppenheimer, L., Stephens, B., Lewis, F.R. and Trunkey, D.D. (1980) Epidemiology of Trauma Deaths. The American Journal of Surgery, 140, 144-150. http://dx.doi.org/10.1016/0002-9610(80)90431-6 